\title{
USO DE IMAGENS PARA DIAGNÓSTICO DE AFECÇÕES OCULARES - REVISÃO DE LITERATURA
}

\author{
Brenda Mendonça de Alcântara ${ }^{1}$ \\ Tryssia Scalon Magalhães Moi ${ }^{1}$ \\ Ivan Ricardo Martinez Padua ${ }^{1}$ \\ Paula Diniz Galera ${ }^{1}$ \\ Gabriela Morais Madruga ${ }^{1}$ \\ Paola Castro Moraes ${ }^{1}$ \\ Isabela Del Ponti ${ }^{2}$
}

\begin{abstract}
RESUMO
A oftalmologia veterinária abrange o diagnóstico das alterações oculares, perioculares e retrobulbares primárias, e também suas manifestações sistêmicas e secundárias, o que se torna possível a partir da avaliação completa do bulbo ocular, órbita e anexos por meio do exame oftálmico completo e de exames complementares. Dentre os exames de imagens que auxiliam no diagnóstico das afecções oculares, a ultrassonografia está entre os exames de imagem mais utilizados na rotina veterinária, auxiliando na observação de anormalidades retrobulbares e na avaliação das estruturas bulbares na presença de opacificação de meios transparentes, por exemplo. Além da ultrassonografia, existem outras modalidades de exames, dentre eles a tomografia computadorizada, ressonância magnética, angiografia, microscopia especular, tomografia de coerência óptica (OCT) e angiografia por tomografia de coerência óptica (OCT-A). Em virtude dos avanços tecnológicos na obtenção de imagens, a avaliação do globo ocular e das estruturas orbitárias pode ser realizada com maior precisão, o que favorece o diagnóstico e a escolha terapêutica adequada. Objetiva-se com esta revisão enumerar os exames disponíveis, suas principais indicações, bem como sua aplicabilidade e estudos em animais domésticos.
\end{abstract}

Palavras-chave: cão, catarata, ecografia, retinopatia, tomografia.

\section{USE OF IMAGES FOR THE DIAGNOSIS OF OCULAR PATHOLOGIES - LITERATURE REVIEW}

\begin{abstract}
Veterinary ophthalmology covers the diagnosis of primary ocular, periocular and retrobulbar alterations, and also their systemic and secondary manifestations. That is possible through the complete clinic evaluation of ocular bulb, orbit and attachments and the use of complementary exams. Among the imaging exams that help in the diagnostics of ocular pathologies, ultrasound is one of the most used exams in the veterinary routine, assisting in the observation of retrobulbar abnormalities and bulbar structures when the transparency of the tissues is committed, for example. There are other modalities of imaging exams like computer tomography, magnetic resonance, angiography, optical coherence tomography (OCT) and angiography optical coherence tomography (OCT-A). Due to technology advances

\footnotetext{
${ }^{1}$ Unesp FCAV - Campus Jaboticabal brealcantara@yahoo.com.br

${ }^{2}$ FCAV Unesp Jaboticabal, *Correspondência editorial: isabeladelponti.idp@gmail.com
} 
in images, the evaluation of ocular globe and orbital structures is more accurate, allowing accurate diagnosis and adequate treatment. The aim of this literature review is list the available exams and their indications, as well as their applicability and studies in domestic animals.

Keywords: cataract, computer tomography, dog, retinopathy, ultrasound.

\section{USO DE IMÁGENES PARA EL DIAGNÓSTICO DE PATOLOGÍAS DE LOS OJOS - REVISIÓN DE LA LITERATURA}

\section{RESUMEN}

La oftalmología veterinaria cubre el diagnóstico de alteraciones oculares primarias, perioculares y retrobulbares, así como sus manifestaciones sistémicas y secundarias, lo que es posible gracias a la evaluación completa del bulbo ocular, la órbita y las inserciones mediante un examen oftálmico completo y exámenes complementarios. Entre las pruebas de imagen que ayudan en el diagnóstico de patologías oculares, la ecografía se encuentra entre las pruebas de imagen más utilizadas en la rutina veterinaria, ayudando en la observación de anomalías retrobulbares y en la evaluación de estructuras bulbosas en presencia de medios transparentes opacos, por ejemplo. Además de la ecografía, existen otros tipos de exámenes, que incluyen tomografía computarizada, resonancia magnética, angiografía, microscopía especular, angiografía, tomografía de coherencia óptica (OCT) y angiografía por tomografía de coherencia óptica (OCT-A). Debido a los avances tecnológicos en la obtención de imágenes, la evaluación del globo ocular y de las estructuras orbitarias se puede realizar con mayor precisión, lo que favorece el diagnóstico y la elección terapéutica adecuada. El objetivo de esta revisión es enumerar las pruebas disponibles, sus principales indicaciones, así como su aplicabilidad y estudios en animales domésticos.

Palabras claves: perro, catarata, ultrasonido, retinopatía, tomografía.

\section{INTRODUÇÃO}

A oftalmologia é um importante ramo da medicina veterinária, sendo frequente a ocorrência de afecções oculares nas espécies domésticas. Inegavelmente, a especialidade vem se desenvolvendo e acompanhando a evolução da oftalmologia médica e as exigências do mercado (1). Os exames complementares, dentre eles os de imagem, são de extrema importância nesse avanço.

A presente revisão de literatura objetiva compilar informações sobre o uso de exames de imagens para o diagnóstico de alterações oculares, abordando exames já existentes na rotina veterinária e também aqueles que ainda estão restritos às pesquisas; tornando a escolha do exame para determinado paciente mais precisa, facilitando assim o diagnóstico e garantindo tratamento eficaz. 


\section{REVISÃO DE LITERATURA}

\section{ULTRASSONOGRAFIA}

A ultrassonografia ocular é importante ferramenta para a oftalmologia veterinária, segmento que vem crescendo a um ritmo constante, fornecendo informações essenciais para prática dessa especialidade (2).

O exame ultrassonográfico ocular, também conhecido como ecografia ocular, caracteriza-se pela rapidez e facilidade de execução em pequenos animais, visto dispensar, muitas vezes, a contenção química dos pacientes $(3,4)$.

É necessário ressaltar sua importância, pois consiste em método não invasivo e em pacientes cujo quadro oftalmológico cursa com opacidade de um dos olhos, inviabilizando a avaliação direta de estruturas intraoculares pelo exame físico, o mesmo permite avaliar a córnea, humor aquoso, lente e humor vítreo $(5,6)$. Na catarata, a qual é necessário avaliar o segmento posterior, bem como do conteúdo intraocular, o ultrassom é de extrema importância, uma vez que permite distinguir enfermidades do segmento posterior e fornece parâmetros da biometria da câmara anterior e do cristalino, os quais auxiliam na programação cirúrgica $(7,8)$.

Outros procedimentos invasivos, como o exame citológico do conteúdo intraocular ou aplicação de fármacos para bloqueio retrobulbar, podem ser assessorados pela ultrassonografia $(6,9,10)$.

Em geral, a ultrassonografia oferece informações como valores biométricos de estruturas, ecotextura e ecogenicidade (11). A imagem é obtida melhor com os transdutores de alta frequência. A escolha do transdutor é fator determinante para o sucesso do exame. Os mais adequados são os transdutores setoriais, microconvexos e lineares. Transdutores de 20 MHz são os mais utilizados na oftalmologia veterinária $(1,12)$.

Dispositivos de separação entre o transdutor e a córnea avaliam melhor a região da câmara anterior e da lente, enquanto o contato direto do transdutor com a córnea permite observação da câmara posterior e do espaço retrobulbar $(12,13)$.

\section{-Métodos Ultrassonográficos Convencionais}

O exame é realizado com o paciente sentado ou em decúbito esternal, sob contenção física delicada, e em muitas das vezes, sem necessidade de sedação ou anestesia geral (4).

Pode ser realizada a técnica transpalpebral, na qual as pálpebras ficam fechadas, mais utilizada em casos de lesões na córnea ou esclera, ou em casos em que as pálpebras, por motivos diversos, impendem o contato direto entre o transdutor e a córnea. Nesta técnica, o paciente tende a permanecer mais colaborativo com o exame, no entanto podem ocorrer artefatos. A outra técnica a ser utilizada é a transcorneal; é realizada com transdutor, coberto por gel acústico estéril para reduzir artefatos e contaminações, diretamente sobre a córnea. Recomenda-se a instilação de colírio anestésico de 60 a 90 segundos antes de iniciar o exame (2).

O gel mais indicado é o gel acústico estéril, sem conservantes ou aromas, solúvel em água e que permita fácil remoção da superfície do olho. Géis a base de celulose devem ser evitados, pois podem gerar irritação na superfície ocular (14). 


\section{- Modo A}

É a modalidade mais utilizada na oftalmologia veterinária. O modo A (figura 1) gera picos de amplitudes que são formados pelas diversas densidades dos tecidos, é o método com maior acurácia, quando comparado ao modo $\mathrm{B}$, principalmente na avaliação de lesões pequenas (5). Em geral, o modo A vem acoplado com modo B nos aparelhos de ultrassonografia ocular, ou seja, há a possibilidade de avaliação pelos dois métodos (A e B). Existem diferentes frequências, que podem ir de 8 a $20 \mathrm{MHz}$. Devido ao movimento do bulbo ocular existe a limitação na interpretação das amplitudes (13).

A biometria das estruturas que são avaliadas é dada pela multiplicação do tempo decorrido pela velocidade do feixe de onda do ultrassom, o qual é calculado pelo próprio aparelho (14).

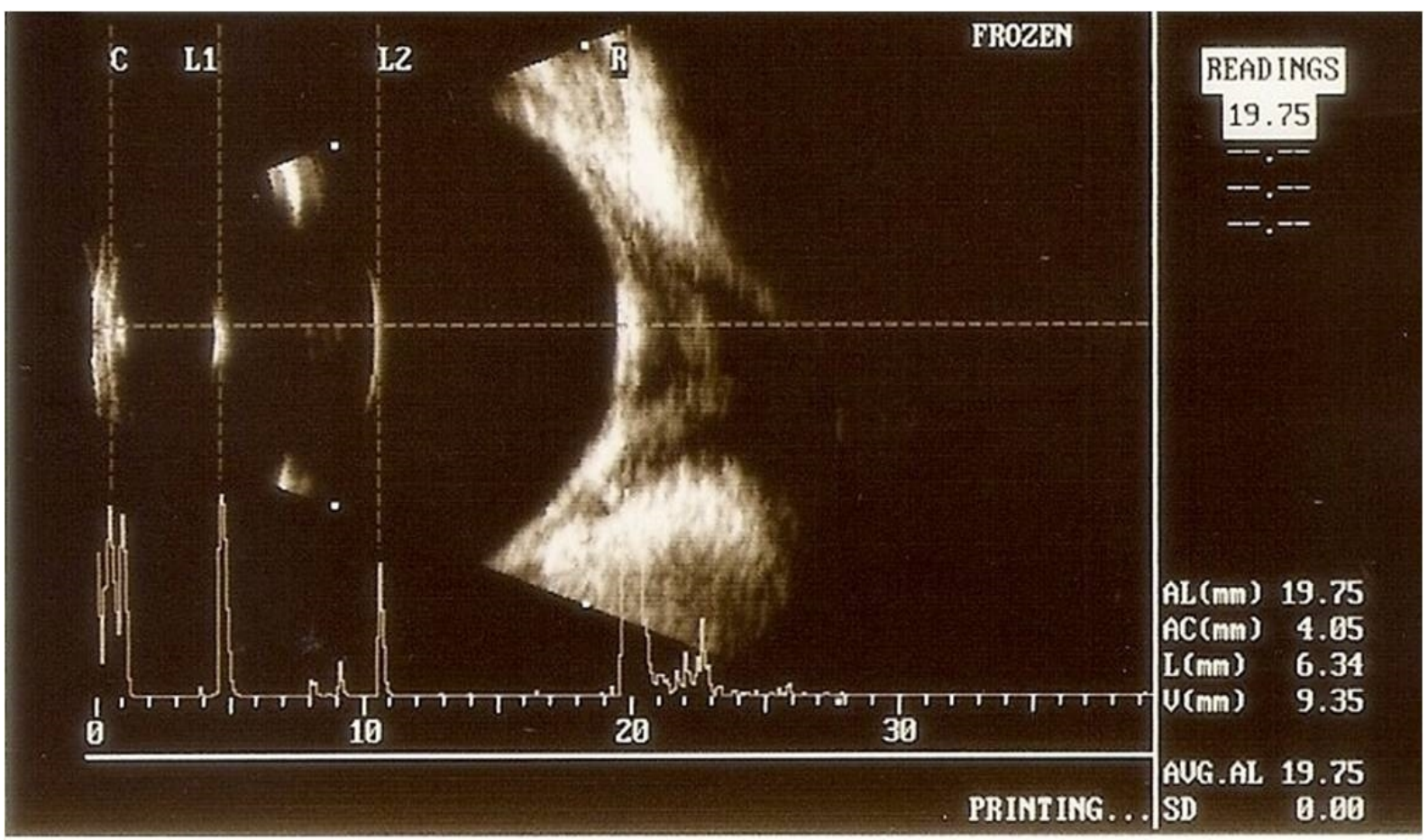

Figura 1. Imagem ultrassonográfica em modos A/B do olho de um cão saudável. Nota-se o aumento na amplitude do traçado correspondente às regiões de endotélio corneal (C), cápsulas anterior (L1) e posterior (L2) da lente e complexo retina-coroideesclera $(\mathrm{R})$. Lateralmente à direita encontram-se as medidas do eixo axial do bulbo ocular (AL), da câmara anterior (AC), da espessura da lente (L) e da câmara vítrea (V). Serviço de Oftalmologia Veterinária - FCAV/Unesp - Jaboticabal.

\section{- Modo B}

Pode ser chamada de modo B ou bidimensional (figura 2), é muito utilizado na oftalmologia veterinária, pois é capaz de detectar contorno e formato das estruturas mesmo quando existe opacidade da córnea (12).

Permite avaliar além da ecogenicidade das estruturas e dos tecidos adjacentes, a forma do tecido e sua biometria. É necessário que haja conhecimento prévio das estruturas oculares na ultrassonografia para identificação das alterações mais sutis (13). 
Para realização do exame é necessário exercer uma compressão mínima do transdutor sobre a córnea, pois em caso de compressão excessiva, esta poderá afetar sua curvatura e alterar os valores reais da câmera anterior. Em alguns casos de perfurações ou úlceras corneais, o exame pode ser contraindicado mesmo pelo método transpalpebral (4).

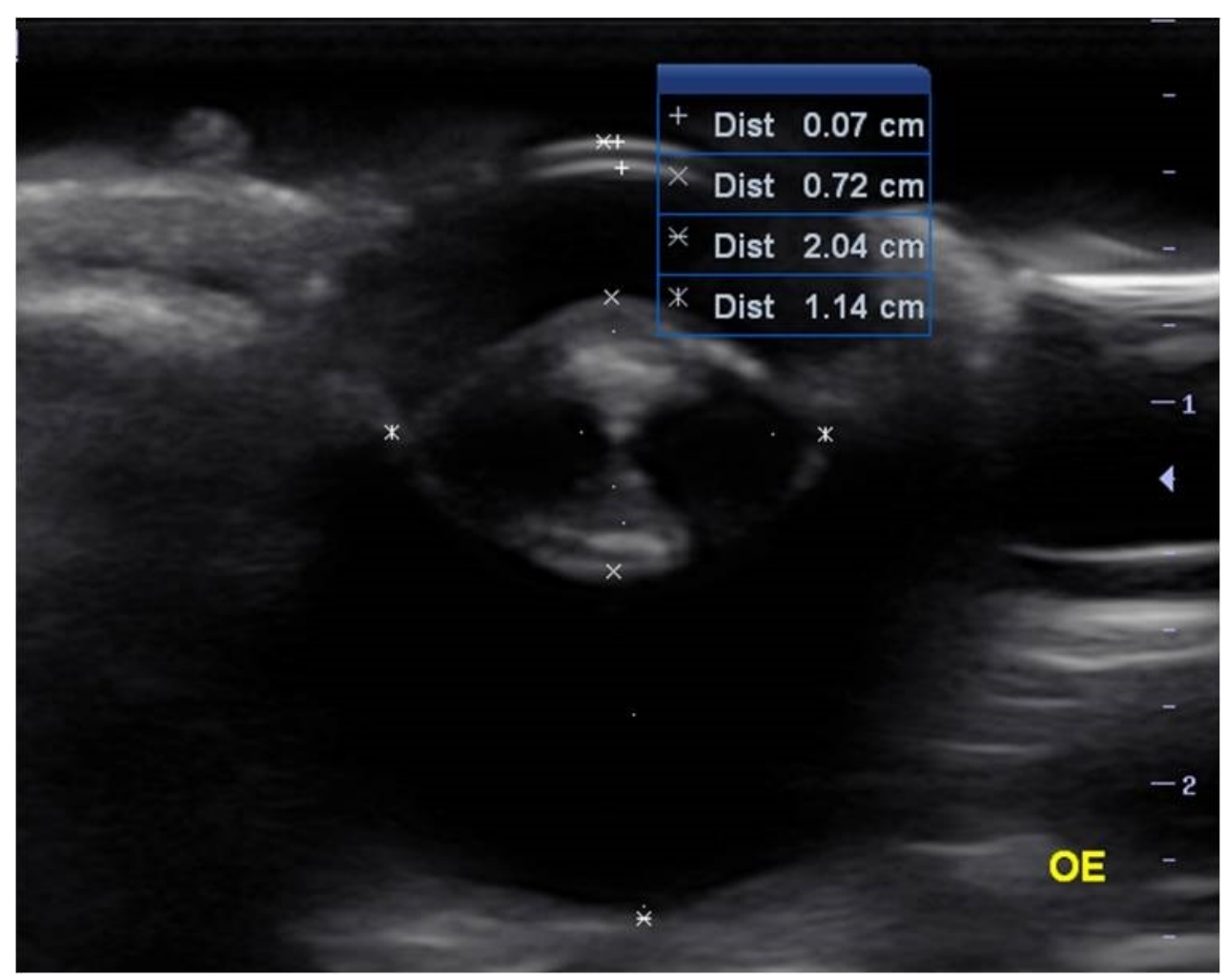

Figura 2. Imagem ultrassonográfica em modo B do olho de um cão com catarata madura. Nota-se dorsalmente linhas hiperecoicas correspondentes ao epitélio e endotélio corneano. Linhas hiperecoicas correspondentes a capsula anterior da lente e posterior da lente, e conteúdo hiperecoico e heterogêneo correspondente a catarata madura (centralmente). Dentro do quadro em azul encontram-se as medidas do eixo axial do bulbo ocular $(1.14 \mathrm{~cm})$, da espessura e comprimento da lente $(0.72 \mathrm{~cm}$; $2.04 \mathrm{~cm})$ e espessura da córnea $(0.07 \mathrm{~cm})$. Serviço de Oftalmologia Veterinária FCAV/Unesp - Jaboticabal.

\section{- Métodos Ultrassonográficos Avançados}

\section{- Elastografia}

Pouco utilizada na oftalmologia veterinária, quando comparada à ultrassonografia tradicional, tem se configurado como útil, ainda que o número de estudos na oftalmologia seja pequeno (15).

A modalidade conhecida como Acoustic Radiation Force Impulse (ARFI) foi utilizada recentemente para avaliar a eficácia do crosslinking em córneas de ratos e de suínos. Em cães, a elastografia ARFI (figura 3) foi utilizada para padronização de imagens de estruturas que compõem o bulbo ocular de raças braquicefálicas (15). 


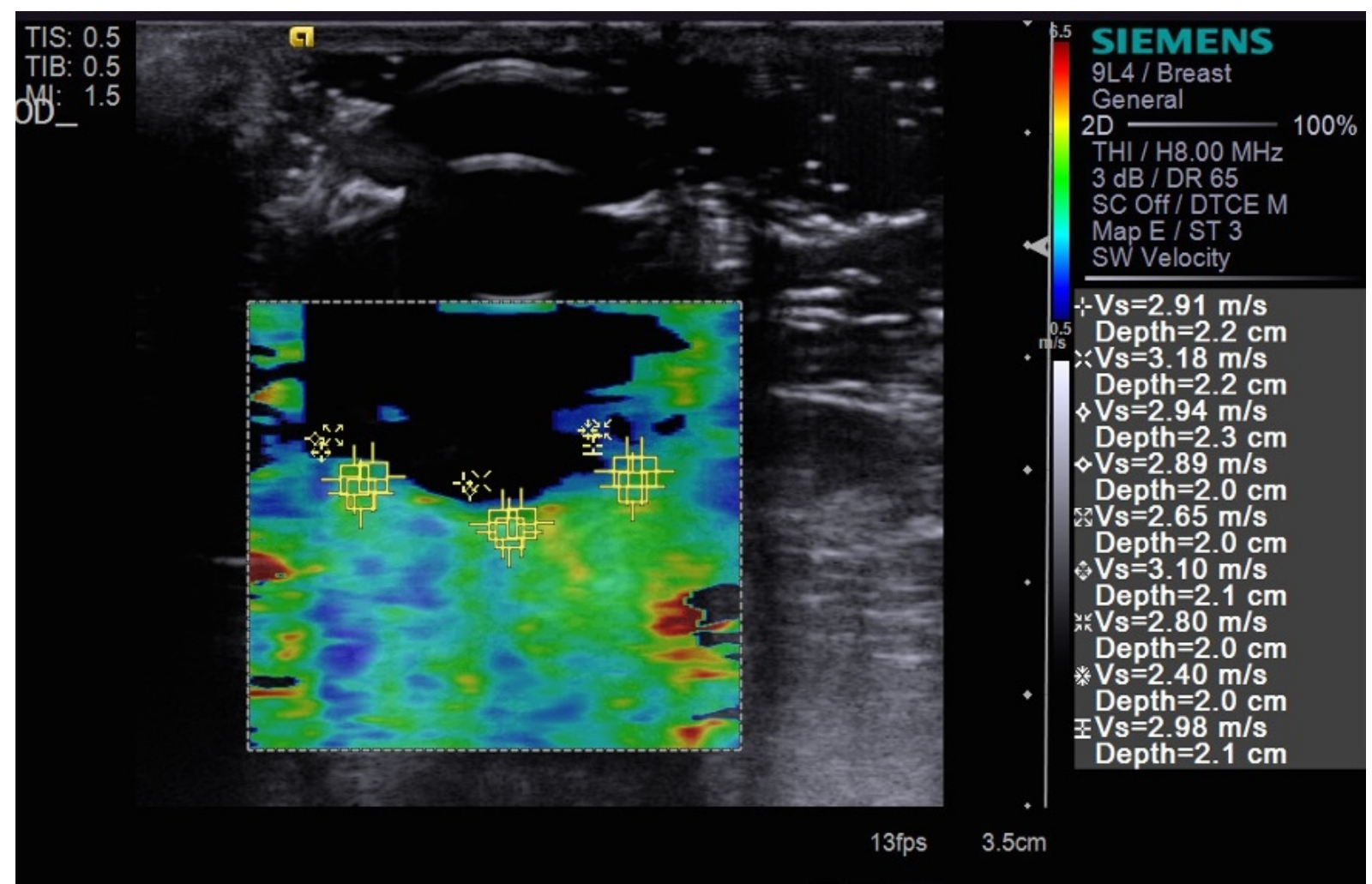

Figura 3. Imagem de elastograma e ARFI quantitativa de um olho de cão saudável. No elastograma qualitativo observa-se estrutura homogênea e macia (tonalidades esverdeadas) em nervo óptico e complexo retina coroide esclera. Lateralmente a direita nota-se a velocidade de cisalhamento dos tecidos.

\section{- Ultrassonografia de contraste por microbolhas}

Para realização da ultrassonografia de contraste por microbolhas (figura 4) utiliza-se substâncias refletoras, administradas ao paciente, que são capazes de intensificar o sinal Doppler, permitindo a detecção de fluxos que em métodos tradicionais são poucos detectáveis. As microbolhas possuem o diâmetro de 1 a $6 \mathrm{~mm}$, podendo permear leitos capilares, leito vascular, mas sem difusão intersticial. Esse método não possui efeitos hemodinâmicos (14).

Na medicina, apresenta bons resultados na avaliação da resposta do paciente acometido por melanoma uveal e submetidos à radiocirurgia. Tem sido objeto de estudo para avaliar a vascularização após a inserção de implantes orbitais de hidroxiapatita, bem como na investigação do padrão vascular em pacientes acometidos por melanoma coroidal, facilitando no diagnóstico precoce da enfermidade. Em animais, sua utilização ainda é experimental, havendo estudos sobre a perfusão uveal em ratos e como método auxiliar na diferenciação entre descolamento de retina e membrana vítrea em cães e gatos (14). 

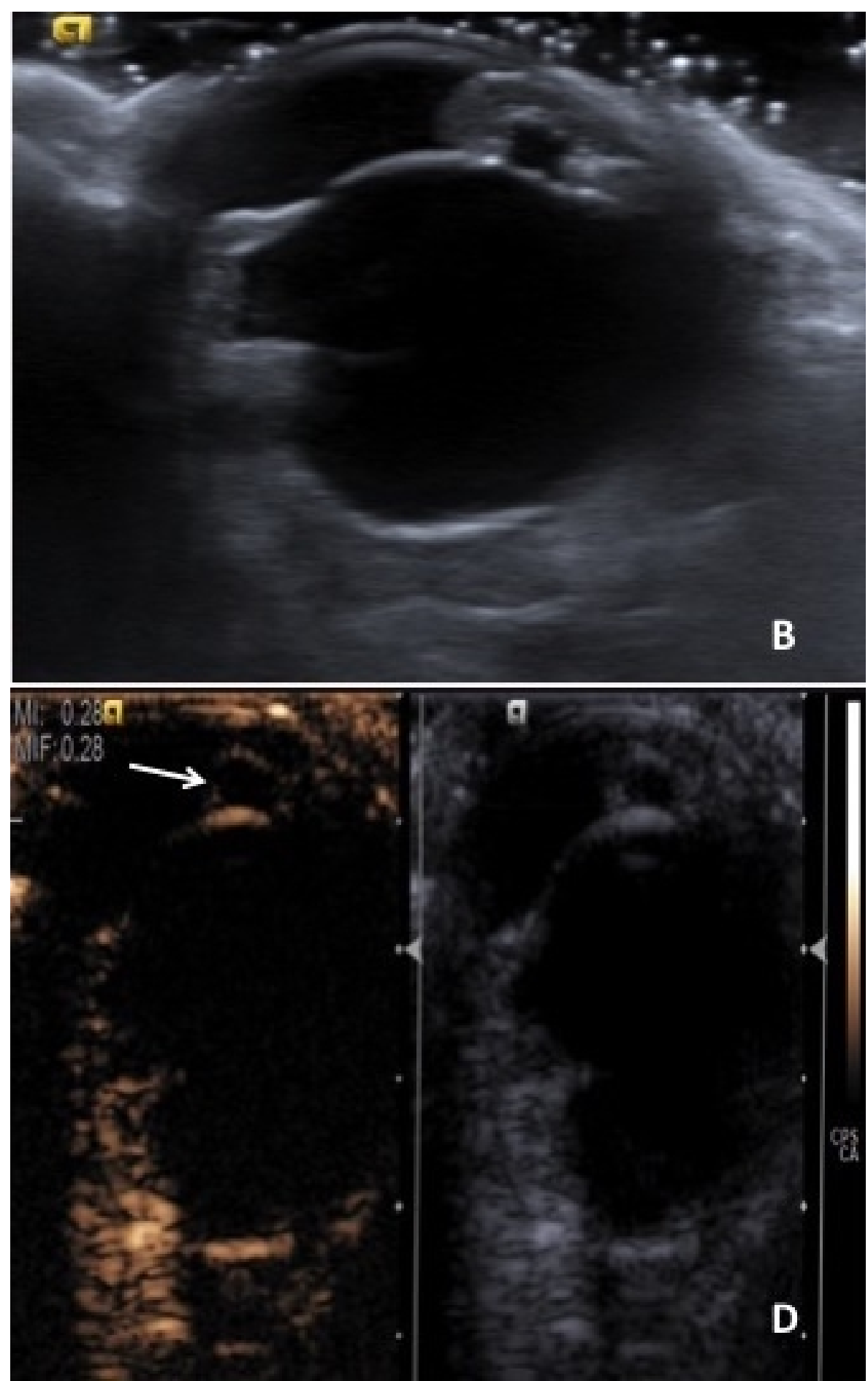

Figura 4 A- Imagem ultrassonográfica em modo B de um gato com uveite. Nota-se aumento de volume e hipercogenicidade da íris. B- Imagem ultrassonográfica com contraste No elastograma qualitativo observa-se estrutura homogênea e macia (tonalidades esverdeadas) em nervo óptico e complexo retina coroide esclera. Lateralmente a direita nota-se a velocidade de cisalhamento dos tecidos. 


\section{- Biomicroscopia ultrassônica}

Esse método possui limitação, pois a penetração das ondas é de apenas 5 a $10 \mathrm{~mm}$. Além de permitir a avaliação detalhada da câmera anterior, é considerada uma das melhores técnicas em diagnóstico por imagem para avaliar estruturas como zônulas lenticulares, corpo ciliar e coroide anterior. Pesquisas comparando a histologia com as imagens da biomicroscopia ultrassônica mostraram que a técnica oferece informações quanto à dimensão das estruturas bem como boa resolução física, permitindo discernir estruturas mesmo quando localizadas muito próximas umas das outras (14).

Em outra pesquisa mostrou-se útil na determinação da patogênese do glaucoma, no diagnóstico precoce do glaucoma de ângulo fechado, na síndrome de efusão ciliar, na subluxação de lente, bem como na identificação de cistos e tumores em corpo ciliar. Em casos de uveítes, podem ser identificados derrame supraciliares e membrana cíclica (14).

\section{- Ultrassonografia modo Doppler}

O método Doppler fornece informações sobre a presença, direção e o fluxo sanguíneo do tecido avaliado. Não permite avaliar o diâmetro do vaso, os valores expressos são sobre a velocidade do fluxo sanguíneo arterial no vaso. O modo Doppler caracteriza-se pela interação do ultrassom modo B com partículas em movimento (16).

Permite avaliar dois tipos de velocidade da onda na artéria, a velocidade sistólica e a velocidade diastólica. O índice de resistência é a medida da interpretação do formato da onda ou do vaso. Resultados com alto índice de resistência podem estar correlacionados com a resistência vascular e a diminuição de perfusão sanguínea (16).

Avaliações funcionais da vascularização ocular, como velocidade do fluxo sanguíneo e resistência vascular, são parâmetros que podem estar alterados em condições patológicas (13).

\section{OUTROS EXAMES DE IMAGENS NA OFTALMOLOGIA VETERINÁRIA}

Na medicina veterinária, além da ultrassonografia, existem outros exames de imagem que auxiliam no diagnóstico de patologias oculares, dentre eles a tomografia computadorizada, ressonância magnética, microscopia especular, angiografia, tomografia de coerência óptica (OCT) e angiografia por tomografia de coerência óptica (OCT-A). A maioria ainda com uso restrito à pesquisa, porém com potencial para fazer parte da rotina clínica.

\section{- Tomografia computadorizada e Ressonância Magnética}

Tanto a tomografia computadorizada quanto a ressonância magnética possuem maior aplicabilidade no diagnóstico de patologias perioculares, destacando-se a órbita, sendo importante também no diagnóstico de corpos estranho e neoplasias.

Devido à sua capacidade de demonstrar as estruturas ósseas de forma minuciosa, a tomografia computadorizada fornece informações precisas sobre todas as estruturas da órbita, possuindo como inconveniente a radiação e a eventual necessidade do uso de contraste (17). Sauvage et al. (18) relataram dois casos de síndrome compartimental da órbita em cães diagnosticados com auxílio da tomografia computadorizada. Enquanto Vieira et al. (19) realizaram avaliação de epífora, na espécie canina, utilizando como um dos métodos a tomografia computadorizada, em comparação com a dacriocistografia.

A ressonância magnética, por sua vez, permite melhor avaliação dos tecidos moles e das vias ópticas, apresentando benefícios na avaliação da extensão da inflamação orbital, por 
exemplo (20). A avaliação de nervos cranianos torna-se possível, como em casos de oftalmoplegia e oftalmoparesia, onde objetiva-se investigar o nervo oculomotor (21).

\section{- Microscopia Especular}

A microscopia especular avalia a camada endotelial da córnea, de forma qualitativa e quantitativa, a qual é responsável pela integridade e transparência corneana. Estudo realizado por Pigatto et al. (22) demonstraram que a morfologia endotelial da córnea de cães é similar à encontrada em humanos.

A análise do endotélio corneal tem aplicabilidade nos pacientes com catarata, visto que o sucesso da facoemulsificação, tratamento de eleição para catarata, depende da celularidade do endotélio. Estudo realizado com aplicação de ácido ascórbico no pós-operatório de facoemulsificação em cães demonstrou, por meio da microscopia especular, que essa substância pode reduzir a perda de células endoteliais da córnea, decorrente do procedimento intraocular (14).

A microscopia especular foi utilizada ainda em estudo comparativo entre pacientes caninos afácicos e pseudofácicos, com intuito de avaliar se a colocação de lente intraocular empregadas no pós-operatório de facoemulsificação interfere na densidade das células endoteliais, o que não foi confirmado com os resultados obtidos (23).

\section{- Angiografia}

A angiografia é um exame que permite avaliar a região de retina, coroide e nervo óptico, baseando-se em imagens seriadas do fundo de olho após injeção de contraste endovenoso. As opções de contraste são fluoresceína e indocianina verde. Em cães, a visualização da coroide fica limitada devido ao tapetum lucidum e pigmentação difusa do fundo de olho nessa espécie, dessa forma Wakaiki et al. (24) realizaram estudo comparativo entre a angiografia com fluoresceína e com indocianina verde, confirmando a hipótese que o exame utilizando indocianina verde mostrou-se mais sensível para avaliação da coroide.

Apesar da angiografia com fluoresceína ser mais comumente utilizada na avaliação do fundo de olho, essa também permite, de forma adaptada, o estudo da câmara anterior, permitindo, por exemplo, a análise da vascularização da íris (25).

\section{- Tomografia de Coerência Óptica (OCT)}

A tomografia de coerência óptica é um exame não invasivo, sem contato, capaz de produzir imagens de alta resolução da retina e do nervo óptico, e é de extrema importância no diagnóstico e resolução de enfermidades do fundo de olho. Cada espécie animal possui suas particularidades em relação ao exame, necessitando de ajustes específicos para garantir imagens de qualidade (26). O exame assemelha-se ao ultrassom no modo B, com a diferença que utiliza luz ao invés do som $(27,28)$.

A posição da cabeça é uma das principais diferenças entre o humano e o cão durante a realização do exame, o que normalmente gera a necessidade de sedação ou anestesia geral do paciente para seu correto posicionamento durante o exame (29).

Safatle et al. (28) realizaram um estudo para comparar os resultados da OCT de animais sadios e animais sabidamente com retinopatias, concluindo que o exame é efetivo no diagnóstico de descolamento de retina e atrofia progressiva de retina. O exame também é indicado na avaliação retiniana de paciente diabéticos, os quais tendem a perder a estratificação da retina, assim como ocorre nos humanos com neuropatia retiniana (30). 
Em cães e gatos, a presença do tapetum lucidum gera a necessidade de adaptação dos parâmetros de escaneamento, visto que há maior espessura retiniana nessa região $(26,28)$.

\section{- Angiografia por Tomografia de Coerência Óptica (OCT-A)}

A angiografia por meio de tomografia de coerência óptica surgiu comercialmente em 2014 e tem como vantagem em relação à angiografia tradicional a análise tridimensional de qualquer espessura ou profundidade da retina e coroide, além de dispensar o uso de contrastes $(31,32)$.

É considerado exame revolucionário na medicina humana, porém ainda em fase de pesquisa na medicina veterinária. Alnawaiseh et al. (33) realizaram estudo em ovinos para analisar alterações na perfusão retiniana diante de um choque hemorrágico, pela OCT-A, concluindo que a mesma diminui consideravelmente e retorna à normalidade após correção da volemia.

\section{CONSIDERAÇÕES FINAIS}

O ultrassom é um dos métodos de diagnóstico por imagem mais utilizados na rotina da oftalmologia veterinária. $\mathrm{O}$ uso dos demais exames no auxílio do diagnóstico de enfermidades oculares enfrenta algumas barreiras na medicina veterinária, dentre elas o custo. Outro empecilho é em relação à anestesia, pois muitas vezes o paciente não está em condições de ser sedado ou anestesiado para execução adequada do exame. O enfoque dado às afecções também norteia a oferta dos métodos diagnósticos, justificando algumas diferenças entre os exames mais utilizados na medicina humana e na veterinária. Apesar das dificuldades, as pesquisas vêm auxiliando na introdução de métodos de diagnóstico avançados na rotina da oftalmologia veterinária.

\section{REFERÊNCIAS}

1. Labruyère JJ, Hartley $\mathrm{C}$, Rogers $\mathrm{K}$, Wetherill $\mathrm{G}$, McConnell JF, Dennis R. Ultrasonographic evaluation of vitreous degeneration in normal dogs. Vet Radiol Ultrasound. 2008;49(2):165-71. doi: https://doi.org/10.1111/j.1740-8261.2008.00344.x.

2. Blohm KO, Hittmair KM, Tichy A, Nell B. Quantitative, noninvasive assessment of intraand extraocular perfusion by contrast-enhanced ultrasonography and its clinical applicability in healthy dogs. Vet Ophthalmol. 2019;1(11):1-11. doi: https://doi.org/10.1111/vop.12648.

3. Boroffka SAEB. Ultrasonographic evaluation of pre- and postnatal development of the eyes in beagles. Vet Radiol Ultrasound. 2005;46(1):72-9. doi: https://doi.org/10.1111/j.1740-8261.2005.00015.x.

4. Galhoefer NS, Bentley E, Ruetten M, Grest P, Haessig M, Kircher PR, et al. Comparison of ultrasonography and histologic examination for identification of ocular diseases of animals: 113 cases (2000-2010). J Am Vet Med Assoc. 2013;243(3):376-88. doi: https://doi.org/10.2460/javma.243.3.376.

5. Dietrich UM. Ophthalmic examination and diagnostics part 3: diagnostic ultrasonography. In: Gellat KN. Veterinary ophthalmology. 4th ed. Oxford: Blackwell; 2007. p. 507-19. 
6. Barsotti G, Mannucci T, Citi S. Ultrasonography-guide dremoval of plant-based foreign bodies from the lacrimal sac in four dogs. BMC Vet Res. 2019;15(1):76. doi: https://doi.org/10.1186/s12917-019-1817-9.

7. Williams DL. Lens morphometry determined by B-mode ultrasonography of the normal and cataractous canine lens. Vet Ophthalmol. 2004;7(2):91-5. doi: https://doi.org/10.1111/j.1463-5224.2004.04005.x.

8. Bhatt AB, Schefler AC, Feuer WJ, Yoo SH, Murray TG. Comparison of predictions made by the intraocular lens masters and ultrasound biometry. Arch Ophthalmol. 2008;126(7):929-33. doi: https://doi.org/10.1001/archopht.126.7.929.

9. Kubal WS. Imaging of orbital trauma. Radiographics. 2008;28(6):1729-39. doi: https://doi.org/10.1148/rg.286085523

10. Luyet C, Eichenberger U, Moriggl B, Remonda L, Greif R. Realtime visualization of ultrasound-guided retrobulbar blockade: an imaging study. $\mathrm{Br} \mathrm{J}$ Anaesth. 2008;101(6):855-9. doi: https://doi.org/10.1093/bja/aen293.

11. Schiffer SP, Rantanen NW, Leary CA, Bryan GM. Biometric study of the canine eye, using A-mode ultrasonography. Am J Anim Vet Sci. 1982;43(5):826-30. doi: https://pubmed.ncbi.nlm.nih.gov/7091846/.

12. Graham KL, Krpckenberger MB, Billson FM. Intraocular sarcoma associated with lens capsule rupture and persistenthy perplastic primary vitreous in a dog. Vet Ophthalmol. 2016;21(2):188-93. doi: https://doi.org/10.1111/vop.12454.

13. Hong S, Park S, Lee D, Cha A, Kim D, Choi J. Contrast-enhanced ultrasonography for evaluation of blood perfusion in normal canine eyes. Vet Ophthalmol. 2019;22(1):31-8. doi: https://doi.org/10.1111/vop.12562.

14. Padua IRM, Abreu TGM, Madruga GM. Olhos In: Feliciano MAR. Ultrassonografia em cães e gatos. São Paulo: Medvet; 2019. p.477- 508.

15. Moon S, Park S, Lee S, Cheon B, Hong S, Cho H, et al. Comparison of elastography, contrast-enhanced ultrasonography, and computed tomography for assessment of lesion margin after radiofrequency ablation in livers of healthy dogs. Am J Anim Vet Sci. 2017;78(3):295-304. doi: https://doi.org/10.2460/ajvr.78.3.295.

16. Novellas R, Espada Y, De Gopegui R. Doppler ultrasonographic estimation of renal and ocular resistive and pulsatility indices in normal dogs and cats. Vet Radiol Ultrasound. 2007;48(1):69-73. doi: https://doi.org/10.1111/j.1740-8261.2007.00206.x

17. Santos AC, Prado PTC, Cruz AAV. Orbital imaging. Arq Bras Oftalmol. 1999;62(2):20819. doi: https://doi.org/10.5935/0004-2749.19990041.

18. Sauvage A, Bolen G, Monclin S, Grauwels M. Orbital compartment syndrome resulting in unilateral blindness in two dogs. Open Vet J. 2018;8(4):445-51. doi: 10.4314/ovj.v8i4.15. 
19. Vieira NMG, Ranzani JJT, Brandão CVS, Cremonini DN, Schellini SA, Padovani CR, et al. Avaliação da epífora em cães usando dacriocistografia e tomografia computadorizada. Pesqui Vet Bras. 2015;35(12):989-96. doi: http://dx.doi.org/10.1590/S0100736X2015001200008.

20. Fischer MC, Busse C, Adrian AM. Magnetic resonance imaging findings in dogs with orbital inflammation. J Small Anim Pract. 2019;60(2):107-15. doi: https://doi.org/10.1111/jsap.12929.

21. Pont RT, Freeman C, Denis R, Hartley C, Beltran E. Clinical and magnetic resonance imaging features of idiopathic oculomotor neuropathy in 14 dogs. Vet Radiol Ultrasound. 2017;58(3):334-43. doi: https://doi.org/10.1111/vru.12478.

22. Pigatto JAT, Cerva C, Freire CD, Abib FC, Bellini LP, Barros SM, et al. Morphological analysis of the corneal endothelium in eyes of dogs using specular microscopy. Pesqui Vet Bras. 2008;28(9):427-30. doi: https://doi.org/10.1590/S0100-736X2008000900006.

23. Nagatsuyu CE, Abreu PB, Kobashigawa KK, Conceição LF, Morales A, Andrade AL, et al. Non-contact specular microscopy in aphakic and pseudophakic dogs. Cienc Rural. 2014;44(4):682-7. doi: http://dx.doi.org/10.1590/S0103-84782014000400018.

24. Wakaiki S, Maehara S, Abe R, Tsuzuki K, Igarashi O, Saito A, et al. Indocyanine green angiography for examining the normal ocular fundus in dogs. J Vet Med Sci. 2007;69(5):465-70. doi: 10.1292/jvms.69.465.

25. Alario AF, Pirie CG, Pizzinari S. Anterior segment fluorescein angiography of the normal canine eye using a dSLR camera adaptor. Vet Ophthalmol. 2013;16(1):10-9. doi: https://doi.org/10.1111/j.1463-5224.2012.01007.x.

26. McLellan GJ, Rasmussen CA. Optical coherence tomography for the evaluation of retinal and optic nerve morphology in animal subjects: practical considerations. Vet Opthalmol. 2012;15 Suppl 2:13-28. doi: https://doi.org/10.1111/j.1463-5224.2012.01045.x.

27. Malerbi FK, Andrade REA, Farah ME. OCT no diagnóstico por imagem. In: Farah ME, editor. Tomografia de Coerência Óptica: OCT. 2a ed. Rio de Janeiro: Cultura Médica, Guanabara Koogan; 2010. p.1-8.

28. Safatle AMV, Braga-Sá MBP, Barros PSM. Aspectos da tomografia de coerência óptica em cães com retinopatia. Pesqui Vet Bras. 2015;35(2):153-9. doi: https://doi.org/10.1590/S0100-736X2015000200010.

29. Hernandez-merino E, Kecova H, Jacobson SJ, Hamouche KN, Nzokwe RN, Grozdanic SD. Spectral domain optical coherence tomography (sd-oct) assessment of healthy female canine retina and optic nerve. Vet Ophthalmol. 2011;14(6):400-5. doi: https://doi.org/10.1111/j.1463-5224.2011.00887.x.

30. Braga-Sá MBP, Barros PSM, Jorge JS, Dongo P, Finkensieper P, Bolzan AA, et al. Retina assessment by optical coherence tomography of diabetic dogs. Braz J Vet Res Anim Sci. 2018;38(10): 1966-71. doi: http://dx.doi.org/10.1590/1678-5150-pvb-5614.

Alcântara BM, Moi TSM, Padua IRM, Galera PD, Madruga GM, Moraes PC, Ponti ID. Uso de imagens para diagnóstico de afecções oculares - Revisão de literatura. Vet. e Zootec. 2021; v28: 001-013 
31. Novais EA, Roisman L, Oliveira PR, Louzada RN, Cole ED, Lane M, et al. Optical coherence tomography angiography of chorioretinal diseases. Ophthalmic Surg Lasers Imaging. 2016;47(9):848-61. doi: https://doi.org/10.3928/23258160-20160901-09.

32. Roisman L. Angiografia por tomografia de coerência óptica: presente e futuro [Internet]. São Paulo: SBVR; 2018 [cited 2019 Mar 3]. Available from: https://www.sbrv.org.br/angiografia-por-tomografia-de-coerencia-optica-presente-efuturo

33. Alnawaiseh M, Etmer C. Seidel L, Arnemann PH, Lahme L, Kampmeier TG, Rehberg $\mathrm{SW}$, et al. Feasibility of optical coherence tomography angiography to assess changes in retinal microcirculation in ovine haemorrhagic shock. Crit Care. 2018;22:138. doi: 10.1186/s13054-018-2056-3.

Recebido em: 16/12/2020

Aceito em: 28/04/2021 